\title{
A Transcriptome Study of Progeroid Neurocutaneous Syndrome Reveals POSTN As a New Element in Proline Metabolic Disorder
}

\begin{abstract}
Yu-Wen Huang ${ }^{1,2}$, Ming-Fu Chiang ${ }^{3,4,5}$, Che-Sheng Ho ${ }^{6}$, Pi-Lien Hung ${ }^{7}$, Mei-Hsin Hsu ${ }^{7}$, Tsung-Han Lee ${ }^{2}$, Lichieh Julie Chu ${ }^{8}$, Hsuan Liu ${ }^{8,9}$, Petrus Tang ${ }^{10}$, Wailap Victor Ng1,11,12, *, Dar-Shong Lin 2,6,13,"

${ }^{1}$ Institute of Biotechnology in Medicine and Department of Biotechnology and Laboratory Science in Medicine, National Yang Ming University, Taipei, Taiwan. ${ }^{2}$ Department of Medical Research, Mackay Memorial Hospital, Taipei, Taiwan. ${ }^{3}$ Department of Neurosurgery, Mackay Memorial Hospital, Taipei, Taiwan. ${ }^{4}$ Mackay Junior College of Medicine, Nursing and Management, Taipei, Taiwan. ${ }^{5}$ Graduate Institute of Injury Prevention and Control, Taipei Medical University, Taipei, Taiwan. ${ }^{6}$ Department of Pediatrics, Mackay Memorial Hospital, Taipei, Taiwan. ${ }^{7}$ Department of Pediatric Neurology, Kaohsiung Chang Gung Memorial Hospital, and Chang Gung University College of Medicine, Kaohsiung, Taiwan. ${ }^{8}$ Molecular Medicine Research Center, Chang Gung University, Taoyuan, Taiwan. ${ }^{9}$ Department of Cell and Molecular Biology, College of Medicine, Chang Gung University, Taoyuan, Taiwan. ${ }^{10}$ Molecular Regulation and Bioinformatics Laboratory and Department of Parasitology, Chang Gung University, Taoyuan, Taiwan. ${ }^{11}$ Institute of Biomedical Informatics and Center for Systems and Synthetic Biology, National Yang Ming University, Taipei, Taiwan. ${ }^{12}$ Department of Biochemistry, Kaohsiung Medical University, Kaohsiung, Taiwan. ${ }^{13}$ Department of Medicine, Mackay Medical College, New Taipei, Taiwan
\end{abstract}

[Received November 27, 2017; Revised February 14, 2018; Accepted February 22, 2018]

\begin{abstract}
Aging is a complex biological process. A study of pyrroline-5-carboxylate reductase 1 (PYCR1) deficiency, which causes a progeroid syndrome, may not only shed light on its genetic contribution to autosomal recessive cutis laxa (ARCL) but also help elucidate the functional mechanisms associated with aging. In this study, we used RNA-Seq technology to examine gene expression changes in primary skin fibroblasts from healthy controls and patients with PYCR1 mutations. Approximately 22 and 32 candidate genes were found to be up- and downregulated, respectively, in fibroblasts from patients. Among the downregulated candidates in fibroblasts with PYCR1 mutations, a strong reduction in the expression of 17 genes $\mathbf{5 3 . 1 \%}$ ) which protein products are localized in the extracellular space was detected. These proteins included several important ECM components, periostin (POSTN), elastin (ELN), and decorin (DCN); genetic mutations in these proteins are associated with different phenotypes of aging, such as cutis laxa and joint and dermal manifestations. The differential expression of ten selected extracellular space genes was further validated using quantitative RT-PCR. Ingenuity Pathway Analysis revealed that some of the affected genes may be associated with cardiovascular system development and function, dermatological diseases and conditions, and cardiovascular disease. POSTN, one of the most downregulated gene candidates in affected individuals, is a matricellular protein with pivotal functions in heart valvulogenesis, skin wound healing, and brain development. Perturbation of PYCR1 expression revealed that it is positively correlated with the POSTN levels. Taken together, POSTN might be one of the key molecules that deserves further investigation for its role in this progeroid neurocutaneous syndrome.
\end{abstract}

Key words: Aging, cutis laxa, progeroid, PYCR1, periostin, ARCL2B

*Correspondence should be addressed to: Dr. Wailap Victor Ng, National Yang Ming University, Taiwan. Email: wvng@ym.edu.tw; Dr. Dar-Shong Lin, Mackay Memorial Hospital, Taipei, Taiwan. Email: dslin@mmh.org.tw

Copyright: () 2018 Huang Y et al. This is an open-access article distributed under the terms of the Creative Commons Attribution License, which permits unrestricted use, distribution, and reproduction in any medium, provided the original author and source are credited. 
Aging is a complex biological process that results in systemic physiological changes, particularly in the skin and the cardiovascular, skeletal, and neuronal systems. Autosomal recessive cutis laxa (ARCL) includes several connective tissue disorder syndromes characterized by abnormal elastic fibers, resulting in sagging, inelastic, and wrinkled skin. It involves multiple organ systems, leading to multisystem disorder characterized by premature aging. It may be used as a human disease model that mimics physiological aging. ARCL type 1 (ARCL1; MIM 219100) is caused by defects in ECM components, and mutations have been identified in the following genes: EGF-containing fibulin-like ECM protein (EFEMP2 or $F B L N 4$ ), fibulin 5 (FBLN5), and latent transforming growth factor-beta-binding protein-4 (LTBP4). Unlike ARCL1, ARCL2 (ARCL2A and ARCL2B; MIM 219200 and MIM 612940) is caused by mutations in genes involved in different metabolic pathways, including glycosylation (ATPase $\mathrm{H}^{+}$transporting V0 subunit A2 (ATP6VOA2); ARCL2A), proline biosynthesis (pyrroline5-carboxylate reductase 1 (PYCR1); ARCL2B), and Golgi apparatus function (RAB6-interacting golgin $(G O R A B)$ ). In addition to connective tissue abnormalities, a spectrum of syndromes with growth and developmental delay, joint laxity, and mental retardation are also found in most ARCL2B patients [1-4]. Previous study showed PYCRI mutations leads to irregular, fragmented, and reduced elastic fibers in the reticular layer of patients skin $[5,6]$. However, the mechanism through which metabolic enzyme deficiency causes connective tissue abnormality is largely unknown.

Proline and its derivative, hydroxyproline, play an essential role in the formation of collagen and elastin. PYCR1 or $\Delta^{1}$-pyrroline-5-carboxylate synthase (P5CS) defect leads to decreased in mitochondrial proline synthesis $[2,7,8]$. PYCR 1 is a member of the PCYR family, which includes PYCR1, PYCR2, and PYCRL. PYCRL is a cytoplasmic enzyme, and PYCR1 and PYCR2 are mitochondrial proteins that catalyze the reduction of $\Delta^{1}$-pyrroline-5-carboxylate to proline, which is the final step in the synthesis of proline from glutamate (Fig. 1A). Moreover, PYCR1 is the most highly expressed in the bone and skin. PYCRl gene mutations may result in ARCL2B. Approximately 60 individuals in more than 30 families have been reported to have PYCR1-related ARCL with progeroid features [5, 6, 9-14]. PYCR1 deficiency leads to a premature aging phenotype, intrauterine growth retardation, a characteristic triangular facial gestalt, psychomotor retardation, hypotonia, and ophthalmologic abnormalities; and progeroid cutaneous manifestations are the most relevant distinctive hallmarks [9]. Although studies have focused on the genotypephenotype correlation, the link between PYCR1 deficiency and connective tissue and ophthalmologic changes remain unexplored.

Studies investigating the clinical applications of nextgeneration sequencing technology have started a new era of molecular genetic diagnosis. The global gene expression profiles of $P Y C R I$ in primary skin fibroblasts from healthy controls and patients with PYCRI mutations were compared using RNA-Seq analysis. Our results provided a comprehensive understanding of how PYCRI mutations may cause connective tissue abnormalities, preglaucoma, and heart valve disease. This work not only helps elucidate the functional mechanisms associated with aging but also provides the first characterization of the transcriptomic basis for PYCR1 mutations causing autosomal recessive neurocutaneous syndrome

\section{MATERIALS AND METHODS}

\section{Patients}

Four patients (P1, P2, P3, and P4), aged 4 to 9 years old, from three families with features of ARCL2B were found to have, PYCRI mutations by DNA sequencing using the BigDye terminator chemistry in this (P4) and our previous (P1, P2, and P3) studies [11, 12]. These patients presented with generalized skin wrinkling, intrauterine growth retardation, a typical facial gestalt, and variable CNS involvement. Consent for molecular studies was given by all individuals involved in this study or their legal representatives. This study was approved by the Ethics Committee of Mackay Memorial Hospital (14MMHIS046).

\section{Cell cultures}

Using standard techniques, skin fibroblasts were isolated and cultured from skin biopsies of the patients and agematched healthy controls (3 to 8 years old). Skin fibroblasts were cultivated in DMEM (Gibco, Bethesda, MD, USA) supplemented with $10 \%$ fetal bovine serum (FBS; Biological Industries, Kibbutz Beit-Haemek, Israel), $1 \%$ glutamine (Gibco), and $1 \%$ penicillin/streptomycin (Gibco) and harvested when the cells reached approximately 100\% confluency (Passage: 6 (RNA-Seq); 6 to 9 (other experiments)).

\section{Transcriptome sequencing}

RNA-Seq analysis were conducted using total RNA samples from two controls and two patients (P1 and P3) isolated with Trizol (Gibco). An aliquot of $2.5 \mu \mathrm{g}$ of total RNA with rRNA removed using Ribo-Zero ${ }^{\mathrm{TM}}$ Gold Kit (Epicentre, Madison, WI, USA) was fragmented with RNase III (Thermo Fisher Scientific, Waltham, MA, 
USA). The yield of fragmented RNA was quantitated using the Quant-iT RNA assay kit (Invitrogen, Carlsbad, CA, USA). After following the protocol for the RiboMinus $^{\text {TM }}$ kit (Thermo Fisher Scientific), 50-ng ribominus RNA fragments were used for transcriptome libraries preparation as per the manufacturer's protocol and were amplified using the $5500 \mathrm{~W}$ Barcode Conversion Primer Kit for 5500 SOLiD System (Life Technologies, Gaithersburg, MD, USA). The bar-coded cDNA libraries were pooled together in equal concentrations and were sequenced in the same quadrant of a slide on a SOLiD sequencer (Applied Biosystems, Foster City, CA, USA) for whole-transcriptome sequencing.

\section{RNA-Seq data analysis}

The high-throughput sequencing of four samples (two healthy controls and two patients, P1 and P3) was performed using single-end, 150 nucleotide reads from the 5500 SOLiD System (Life Technologies). All quality color-space reads were cut to 50 nucleotides and mapped against the NCBI Build 37/hg19 genome using LifeScope Genome Analysis Software v2.5.1 with default RNA-Seq parameters (Life Technologies). Expression values of each gene in TPM (transcripts per million) were determined and statistical analysis performed in the RNASeq workflow of Partek Genomics Suite (v6.6, St. Louis, MO, USA). Gene Ontology and Function analysis were performed using the PANTHER Classification System (www.pantherdb.org/) $[15,16]$ and Ingenuity ${ }^{\circledR}$ Pathway Analysis (IPA) software (www.ingenuity.com), respectively.

\section{Quantitative RT-PCR (qRT-PCR)}

Fibroblast total RNAs were isolated using an RNeasy Mini Kit (QIAGEN, Hilden, Germany) according to the manufacturer's procedure. Complementary DNAs were synthesized by the reverse transcription of $1 \mu \mathrm{g}$ of total RNAs by using a RevertAid H Minus First Strand cDNA Synthesis Kit (Thermo Fisher Scientific). qPCR was performed using a Fast SYBR ${ }^{\circledR}$ Green Master Mix (Applied Biosystems) on an ABI Prism 7500 (Applied Biosystems) with gene-specific primers (Supplementary Table 2). For data analysis, raw threshold cycle (CT) values were normalized to $G A P D H$ to obtain $\triangle \mathrm{CT}$. Normalized $\triangle \mathrm{CT}$ was calibrated to the control cell samples for $\Delta \Delta \mathrm{CT}$.

\section{Proline assay}

Fibroblasts cultured in DMEM containing 5\% FBS for 3 days were harvested and lyzed through sonication after resuspension in $1 \mathrm{X}$ PBS. The cell lysates were centrifuged at $15,000 \mathrm{~g}$ at $4^{\circ} \mathrm{C}$ for $10 \mathrm{~min}$, and the proteins in the supernatant were depleted by incubation with a final concentration of $10 \%$ trichloroacetic acid on ice for $5 \mathrm{~min}$ and were centrifuged as described earlier. The proline concentration in the protein-depleted supernatant was determined according to the method of Wang et al. [17]. Briefly, $0.5 \mathrm{~mL}$ each of glacial acetic acid and Chinard's reagent $(2.5 \mathrm{~g}$ of ninhydrin dissolved in $60 \mathrm{~mL}$ of glacial acetic and $40 \mathrm{~mL}$ of $6 \mathrm{M}$ orthophosphoric acid at $70^{\circ} \mathrm{C}$ ) were added to $250 \mu \mathrm{L}$ of the supernatant and incubated for $10 \mathrm{~min}$ at $90^{\circ} \mathrm{C}$. The proline concentration was determined colorimetrically at $515 \mathrm{~nm}$ and was calculated using the proline calibration curve.

\section{Western blot analysis}

An aliquot of $20 \mu \mathrm{g}$ of proteins was separated on $10 \%$ or $12 \%$ SDS-PAGE and transferred to PVDF membranes (Amersham-Pharmacia Biotech Inc., Buckinghamshire, UK). After the membranes were blocked with 5\% skim milk in TBST buffer $(25 \mathrm{mM}$ Tris-HCl, $137 \mathrm{mM} \mathrm{NaCl}$, and $0.1 \%$ Tween $20, \mathrm{pH} 7.4$ ) for $1 \mathrm{~h}$, the membranes were incubated with rabbit anti-human PYCR1(Cat. no. 131081-AP), PYCR2 (Cat. no. 17146-1-AP) (Proteintech), and POSTN (Cat. no. ab152099, Abcam) polyclonal antibodies, and mouse anti $\beta$-actin (Cat no. A2228) and $\alpha$ tubulin (Cat. no. T6199) (Sigma) monoclonal antibodies at $4{ }^{\circ} \mathrm{C}$ overnight. After the membranes were washed three times with TBST, the membranes were incubated with horseradish peroxidase (HRP)-conjugated [goat antirabbit or -mouse] IgG secondary antibody (1: 5,000-1: $10,000)$ at room temperature for $1 \mathrm{~h}$, and the immune reactions were detected using an ECL detection system (Immobilon Western Chemiluminescent HRP Substrate, Millipore, MA, USA).

\section{Knockdown of PYCR1 in fibroblasts}

Five recombinant shRNAs for the PYCRI gene (shPYCR1 clone IDs: TRCN0000038979, -80, -81, -82, and -83 ) and TRC1 obtained from the National RNAi Core Facility at Academia Sinica, Taipei, Taiwan were subcloned into the pLKO plasmid to obtain shPYCR1 \#1 to \#5 and the scramble control. For silencing PYCR1 in primary skin fibroblasts, shPYCR1-\#1-\#5 lentiviruses were generated by transfecting $293 \mathrm{~T}$ cells with the recombinant DNAs and the lentiviral helper plasmids by using the TransIT-LT1 in vitro transfection reagent (Mirus, Madison, WI, USA). After removing the cell debris in the supernatant by centrifugation according to the recommended procedure, lentiviruses were harvested $48 \mathrm{~h}$ post-transfection by filtration through $0.22-\mu \mathrm{m}$ cellulose acetate filters. Subsequently, skin fibroblasts 
$\left(10^{5}\right.$ cells/well in a 6 -well plate) were cultured in a medium containing $8 \mu \mathrm{g} / \mathrm{mL}$ polybrene, infected with the lentiviruses (MOI $=2$ or 5), and incubated at $37^{\circ} \mathrm{C}$ with $5 \% \quad \mathrm{CO}_{2}$ according to the recommended procedure. Selection of the infected cells began on day 3 with medium containing $1 \mu \mathrm{g} / \mathrm{mL}$ puromycin, which was replaced every 3 days.

\section{Overexpression of PYCR1 in fibroblasts}

The PYCR1 overexpression plasmid was constructed by cloning a 1001-bp cDNA fragment, which consisted of the 960-bp CDS of PYCRI (Acc. no. NM_001282280), and $B a m \mathrm{HI}$ and EcoRI adaptors, into a lentiviral vector pCDH-CMV-MCS-EF1-Puro (System Biosciences,
Mountain View, CA). The cDNA fragment was prepared by PCR amplification of a cDNA library originating from healthy human skin fibroblasts with forward and reverse primers (Supplementary Table 1) by using the highfidelity Phusion enzyme (New England Biolabs, Ipswich, MA). Preparation of lentiviral particles and transfection of skin fibroblasts ( $\mathrm{MOI}=2,5$, or 30 ) were conducted as described earlier. PYCR1 overexpression was verified through real-time PCR and Western blotting.

\section{Statistical analysis}

Data are reported as means and standard deviations (SDs) unless indicated otherwise. The significance of pairwise comparisons was determined by Student's t-test.

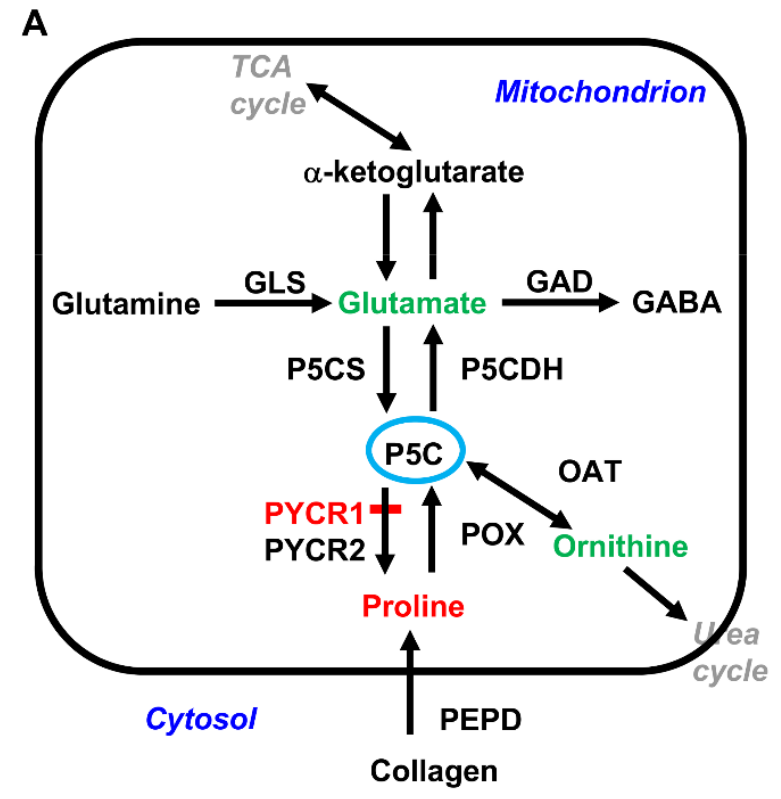

B

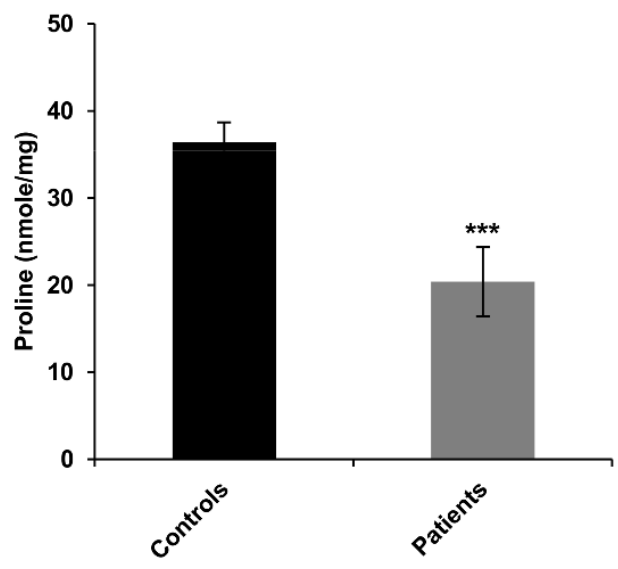

Figure 1. Intracellular proline levels of ARCL2B patient primary skin fibroblasts are lower than healthy controls. (A) Overview of proline biosynthesis. Proline is synthesized via glutamate and ornithine routes. The enzymes P5CS and OAT catalyze the conversion of glutamate and ornithine, respectively, to P5C which is subsequently converted to proline by PYCR1 and PYCR2. (B) Intracellular proline levels in the fibroblasts from healthy controls and patients with PYCR1 mutations (n=4). P5C: $\Delta^{1}$-pyrroline-5-carboxylate; GLS: Glutaminase; GAD: Glutamate decarboxylase; GABA: $\gamma$ - aminobutyric acid; P5CS: P5C synthase; P5CDH: P5C dehydrogenase; OAT: Ornithine aminotransferase; POX: Proline oxidase; PEPD: Proline dipeptidase; $* * *: p<0.001$

\section{RESULTS}

\section{Clinical features of ARCL2B individuals with PYCR1 mutations}

The clinical features of four affected individuals (P1 to P4) from three families are listed in Table 1. DNA sequencing analysis of DNA from bloods of patients and parents identified three mutations in PYCRl gene. Two of the patients (P2 and P3) had a common homozygous single-base deletion (c.345delC) in exon 4, leading to a frame shift and premature termination of translation (p.P115fsX7) of PYCR1. The other two patients had compound heterozygous mutations, including the same single-base deletion (c.345delC) and the missense 743Gto-A (P1) and 559G-to-A (P4) transitions in PYCR1. The 
clinical features of patients $\mathrm{P} 1, \mathrm{P} 2$ and $\mathrm{P} 3$ have been reported in our previous papers $[11,12]$. Patient $\mathrm{P} 4$, a 4year-old male, was the only child of healthy parents. He was born by cesarean section at 38 weeks gestation following an uneventful pregnancy. His birth weight (2.6 $\mathrm{kg}, 10 \mathrm{th}$ percentile) and length (46 $\mathrm{cm}, 4$ th percentile) were at the lower range of norm, while his head circumference $(30.5 \mathrm{~cm})$ was less than the third percentile. Physical examination at birth revealed prominent forehead, wide fontanels, fine and sparse hair, blue sclera, strabismus, back placed and large helices, wrinkled and translucent skin with prominent superficial venous reticule over the trunk and root of limbs, and hypotonia. Both the karyotyping analysis and sonographies of the brain and abdominal organs at birth did not reveal any abnormalities. He had dislocation of left hip joints and bilateral inguinal hernia which were surgical corrected at the age of 8 months and 2 years, respectively. Development milestones were delayed that he began to walk at the age of 1 year and 2 months and could still spoke only few words at the age of 2 years.

Table 1. Clinical features manifested in four ARCL2B patients with PYCRl mutations.

\begin{tabular}{|c|c|c|c|c|c|}
\hline & \multirow{2}{*}{$\begin{array}{l}\text { Family } \\
\text { Patient ID }\end{array}$} & \multirow{2}{*}{$\begin{array}{c}1 \\
\mathrm{P} 1\end{array}$} & \multicolumn{2}{|c|}{2} & \multirow{2}{*}{$\begin{array}{c}3 \\
\mathrm{P} 4\end{array}$} \\
\hline & & & $\mathrm{P} 2$ & P3 & \\
\hline & Gender & Male & Male & Female & Male \\
\hline & Age & 6 & 9 & 8 & 4 \\
\hline \multirow{6}{*}{ 気 } & Status & Heterozygous & Homozygous & Homozygous & Heterozygous \\
\hline & Exon & $4 \& 6$ & 4 & 4 & $4 \& 5$ \\
\hline & CDS & c. 345 delC & c. $345 \mathrm{delC}$ & c. $345 \mathrm{delC}$ & c. $345 \mathrm{delC}$ \\
\hline & & c. $743 \mathrm{G}>\mathrm{A}$ & & & c. $559 \mathrm{G}>\mathrm{A}$ \\
\hline & Protein & p.P115fsX7 & p.P115fsX7 & p.P115fsX7 & p.P115fsX7 \\
\hline & & p.G248E & P.Г IIJISA & p.PIIJISX & p.A187T \\
\hline \multirow{20}{*}{ 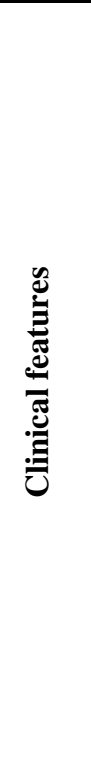 } & Lax wrinkled skin & + & + & + & + \\
\hline & Typical facial gestalt & + & + & + & + \\
\hline & Hernias & + & + & - & + \\
\hline & Preglaucoma & + & ND & ND & + \\
\hline & Cataract & + & - & - & + \\
\hline & Esotropia & + & ND & ND & + \\
\hline & Aortic root dilatation & ++ & - & - & + \\
\hline & Sinus of Valsalva (mm) & 23.6 & 19.8 & 19.1 & 22.5 \\
\hline & (Range) & $(13.56-20.81)$ & $(14.16-21.71)$ & $(16.59-25.55)$ & $(14.05-21.54)$ \\
\hline & Z-Score & 3.47 & 0.97 & -0.86 & 2.46 \\
\hline & Cardiovascular system & IRBBB & MR, PR & TR, MR, RAA & $\mathrm{TR}, \mathrm{AR}$ \\
\hline & Osteopenia & + & + & + & + \\
\hline & Joint hyperlaxity & + & + & + & + \\
\hline & Adducted thumb & + & + & + & + \\
\hline & Hip dislocation & + & + & + & + \\
\hline & Shoulder dislocation & - & + & + & - \\
\hline & Abnormal brain MRI & - & - & - & - \\
\hline & Mental retardation & ++ & ++ & + & $+/-$ \\
\hline & Autism & + & + & - & - \\
\hline & Athetoid movements & + & ++ & - & - \\
\hline \multirow{6}{*}{ 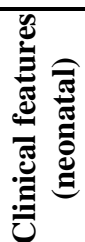 } & Thin, translucent skin & + & + & + & + \\
\hline & Postnatal growth delay & + & + & + & - \\
\hline & Hypotonia & + & + & + & + \\
\hline & Late fontanel closure & + & + & + & + \\
\hline & Blue sclera & + & + & + & + \\
\hline & Strabismus & + & - & - & + \\
\hline
\end{tabular}

IRBBB: incomplete right bundle branch block; MR: mitral regurgitation; PR: pulmonary regurgitation; TR: tricuspid regurgitation; RAA: right aortic arch pressure gradient; AR: aortic regurgitation. ND, not done; -, absent; $+/-$ mild; + , moderate; ++ , severe. 
Table 2. List of 54 differentially expressed gene candidates in ARCL2B patients' primary skin fibroblasts.

\begin{tabular}{|c|c|c|c|c|}
\hline Gene Symbol & Gene Description & $\begin{array}{l}\text { Fold } \\
\text { Change } \\
\text { (Patients/ }^{\text {Controls) }}\end{array}$ & Subcellular Location & Functional Type \\
\hline \multicolumn{5}{|c|}{ Genes downregulated in patients } \\
\hline POSTN & Periostin & -17.22 & Extracellular space & Other \\
\hline$M M P 1$ & Matrix metallopeptidase 1 & -12.22 & Extracellular space & Peptidase \\
\hline PTGS2 & Prostaglandin-endoperoxide synthase 2 & -9.64 & Cytoplasm & Enzyme \\
\hline EFEMP1 & $\begin{array}{l}\text { EGF containing fibulin like } \\
\text { extracellular matrix protein } 1\end{array}$ & -8.69 & Extracellular space & Enzyme \\
\hline$I G F B P 3$ & $\begin{array}{l}\text { Insulin like growth factor binding } \\
\text { protein } 3\end{array}$ & -4.44 & Extracellular space & Other \\
\hline$J A G 1$ & Jagged 1 & -4.37 & Extracellular space & Growth factor \\
\hline$M Y O 1 D$ & Myosin ID & -4.27 & Cytoplasm & Enzyme \\
\hline$E L N$ & Elastin & -3.76 & Extracellular space & Other \\
\hline$C 1 S$ & Complement $\mathrm{C} 1 \mathrm{~s}$ & -3.61 & Extracellular space & Peptidase \\
\hline$D C N$ & Decorin & -3.45 & Extracellular space & Other \\
\hline$C Y P 1 B 1$ & $\begin{array}{l}\text { Cytochrome p } 450 \text { family } 1 \text { subfamily B } \\
\text { member } 1\end{array}$ & -3.43 & Cytoplasm & Enzyme \\
\hline PTX3 & Pentraxin 3 & -3.35 & Extracellular space & Other \\
\hline STK32B & Serine/threonine kinase 32B & -3.26 & Other & Kinase \\
\hline$C 1 R$ & Complement $\mathrm{C} 1 \mathrm{r}$ & -3.11 & Extracellular space & Peptidase \\
\hline$F B L N 2$ & Fibulin 2 & -3.06 & Extracellular space & Other \\
\hline$A D A M 12$ & ADAM metallopeptidase domain 12 & -2.96 & Plasma membrane & Peptidase \\
\hline SPOCK1 & $\begin{array}{l}\text { SPARC/osteonectin, cwcv and kazal } \\
\text { like domains proteoglycan } 1\end{array}$ & -2.95 & Extracellular space & Other \\
\hline$C E B P D$ & CCAAT/enhancer binding protein delta & -2.85 & Nucleus & Transcription regulator \\
\hline COL3A1 & Collagen type III alpha 1 chain & -2.54 & Extracellular space & Other \\
\hline ADAMTS5 & $\begin{array}{l}\text { ADAM metallopeptidase with } \\
\text { thrombospondin type } 1 \text { motif } 5\end{array}$ & -2.51 & Extracellular space & Peptidase \\
\hline$L G R 4$ & $\begin{array}{l}\text { Leucine rich repeat containing } \mathrm{G} \\
\text { protein-coupled receptor } 4\end{array}$ & -2.44 & Plasma membrane & Transmembrane receptor \\
\hline$A R L A C$ & $\begin{array}{l}\text { ADP ribosylation factor like GTPase } \\
4 C\end{array}$ & -2.43 & Nucleus & Enzyme \\
\hline ACKR4 & Atypical chemokine receptor 4 & -2.38 & Plasma membrane & G-protein coupled receptor \\
\hline EMILIN2 & Elastin microfibril interfacer 2 & -2.30 & Extracellular space & Other \\
\hline$J U N B$ & $\begin{array}{l}J U N B \text { proto-oncogene, } \mathrm{AP}-1 \\
\text { transcription factor subunit }\end{array}$ & -2.28 & Nucleus & Transcription regulator \\
\hline NREP & Neuronal regeneration related protein & -2.25 & Cytoplasm & Other \\
\hline$N N M T$ & Nicotinamide N-methyltransferase & -2.14 & Cytoplasm & Enzyme \\
\hline$A L P K 2$ & Alpha kinase 2 & -2.13 & Nucleus & Kinase \\
\hline$L M C D 1$ & LIM and cysteine rich domains 1 & -2.09 & Cytoplasm & Transcription regulator \\
\hline$N P R 3$ & Natriuretic peptide receptor 3 & -2.07 & Plasma membrane & G-protein coupled receptor \\
\hline VEGFA & Vascular endothelial growth factor A & -2.05 & Extracellular space & Growth factor \\
\hline THBS2 & Thrombospondin 2 & -2.03 & Extracellular space & Other \\
\hline \multicolumn{5}{|c|}{ Genes upregulated in patients } \\
\hline HOXB7 & Homeobox B7 & 23.53 & Nucleus & Transcription regulator \\
\hline STMN2 & Stathmin 2 & 10.52 & Plasma membrane & Other \\
\hline TRHDE & $\begin{array}{l}\text { Thyrotropin releasing hormone } \\
\text { degrading enzyme }\end{array}$ & 7.12 & Plasma membrane & Peptidase \\
\hline$S P P 1$ & Secreted phosphoprotein 1 & 3.66 & Extracellular space & Cytokine \\
\hline EPDRI & Ependymin related 1 & 3.54 & Nucleus & Other \\
\hline
\end{tabular}




\begin{tabular}{|c|c|c|c|c|}
\hline$L B H$ & Limb bud and heart development & 3.04 & Nucleus & Transcription regulator \\
\hline$Q P R T$ & Quinolinate phosphoribosyltransferase & 2.92 & Cytoplasm & Enzyme \\
\hline CRIP2 & Cysteine rich protein 2 & 2.79 & Nucleus & Other \\
\hline CRIP1 & Cysteine rich protein 1 & 2.68 & Cytoplasm & Other \\
\hline MEIS1 & Meis homeobox 1 & 2.58 & Nucleus & Transcription regulator \\
\hline PRLR & Prolactin receptor & 2.46 & Plasma membrane & Transmembrane receptor \\
\hline$C B R 3$ & Carbonyl reductase 3 & 2.37 & Cytoplasm & Enzyme \\
\hline PMAIP1 & $\begin{array}{l}\text { Phorbol-12-myristate-13-acetate- } \\
\text { induced protein } 1\end{array}$ & 2.33 & Cytoplasm & Other \\
\hline$P T N$ & Pleiotrophin & 2.28 & Extracellular space & Growth factor \\
\hline ATF5 & Activating transcription factor 5 & 2.20 & Nucleus & Transcription regulator \\
\hline CSPG4 & Chondroitin sulfate proteoglycan 4 & 2.20 & Plasma membrane & Other \\
\hline SH2D5 & SH2 domain containing 5 & 2.19 & Plasma membrane & Other \\
\hline$A M I G O 2$ & $\begin{array}{l}\text { Adhesion molecule with Ig like domain } \\
2\end{array}$ & 2.18 & Plasma membrane & Other \\
\hline CNIH3 & $\begin{array}{l}\text { Cornichon family ampa receptor } \\
\text { auxiliary protein } 3\end{array}$ & 2.17 & Plasma membrane & Transporter \\
\hline HISTIH4H & Histone cluster $1 \mathrm{H} 4$ family member $\mathrm{h}$ & 2.15 & Nucleus & Other \\
\hline$A N K R D 30 B L$ & Ankyrin repeat domain 30B like & 2.11 & Other & Other \\
\hline ADGRE5 & $\begin{array}{l}\text { Adhesion g protein-coupled receptor } \\
\text { E5 }\end{array}$ & 2.08 & Plasma membrane & G-protein coupled receptor \\
\hline
\end{tabular}

At the time of our assessment at 4 years old, patient P4 has achieved weight $(19.6 \mathrm{~kg})$ and height $(110.5 \mathrm{~cm})$ catch-up. Clinical assessment demonstrated features of prominent forehead, large helices, protruding ears, hypermobility of small joints of the hands and feet, and wrinkled and inelastic skin with prominent veins over the dorsum of the hands and feet. The ophthalmic examination revealed preglaucoma, estropia, amblyopia, astigmatism and mild vitreous opacities. Brain MRI revealed no abnormality, while echocardiographic evaluation revealed dilated aortic root with Z-score of 2.46 [18] and skeletal radiography revealed scoliosis. Psychological assessment revealed poor attention and mild intellectual disability with full scale intellectual quotient of 69 at age of 4 years. He received cataract surgery in the right eye at age of 5 years and in the left eye at 8 years.

PYCR1 deficiency leads to a premature aging phenotype in multiple systems, including vision (preglaucoma and cataract), cardiovascular system (aortic root dilatation and valve regurgitation), bone (osteopenia, joint hyperlaxity, and hip dislocation), central nervous system (CNS; mental retardation, autism, and athetoid movements), typical triangular facial gestalt, and progeroid cutaneous manifestations $[5,6,10-12]$. Notably, all four patients had cardiovascular diseases, which has not been reported before.

\section{PYCRI mutations associated with reduced proline level and PYCR1 expression in primary skin fibroblasts}

PYCR1 catalyzes the final step in proline synthesis, in which pyrroline-5-carboxylate is converted to proline in the mitochondria; thus, PYCR1 defects may affect proline synthesis (Fig. 1A). Examination of primary skin fibroblasts revealed that patients with PYCRI mutations had lower intracellular proline levels (Fig. 1B). The truncated PYCR1 protein p.P115fsX7 was not detectable in fibroblasts from patients $\mathrm{P} 2$ and $\mathrm{P} 3$ with homozygous c. 345 delC mutations as revealed by Western blotting, whereas different levels of p.G248E and p.A187T mutant proteins, but not the truncated form, were found in fibroblasts from patients $\mathrm{P} 1$ and $\mathrm{P} 4$ with heterozygous PYCR1 mutations, respectively (Fig. 2A and 2B). The observed differences between patients with homozygous and heterozygous PYCRI mutations indicated that the premature termination codons resulting from the c.345delC mutation might have caused the downregulation of PYCRI mRNA due to nonsensemediated mRNA decay, and that the amino acid substitutions might have affected protein stability. Furthermore, pulse-chase analysis indicated that the PYCR1 p.G248E mutant protein showed a slower degradation rate than the p.A187T mutant protein (Fig. 2C and 2D). In addition, patient with the p.G248E mutant protein had more severe neurological and orthopedic manifestations, suggesting that p.G248E affects protein function. 

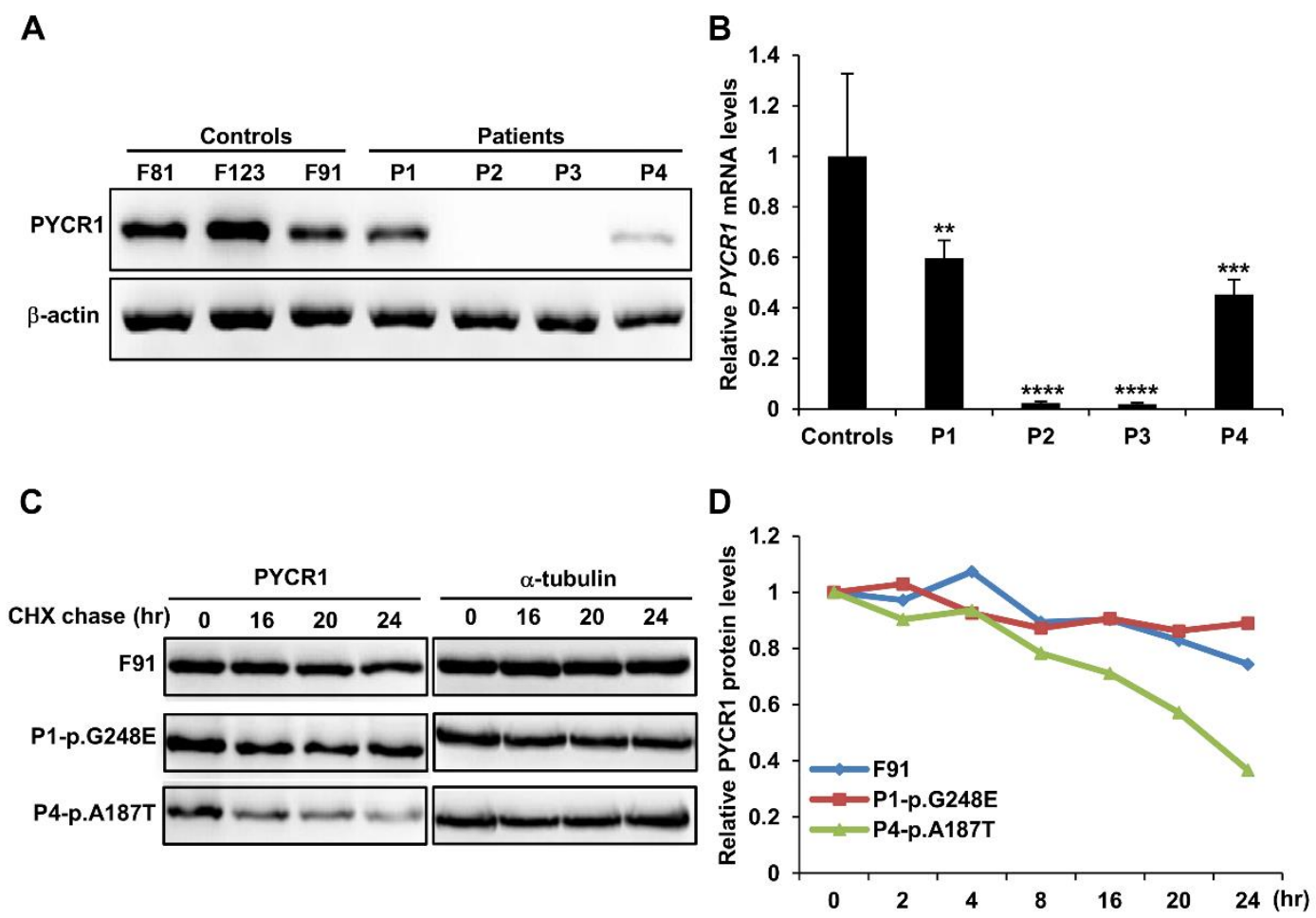

\begin{abstract}
Figure 2. PYCR1 mutations affected its mRNA and protein levels in patients' primary skin fibroblasts. (A) Immunoblot showing the truncated PYCR1 protein p.P115fs X7 is undetectable in fibroblasts from patients (P2 and P3) with homozygous c.345delC mutation, whereas the mutant proteins p.G248E (P1) and p.A187T (P4) are detected in samples with heterozygous mutations. (B) PYCRI levels relative to GAPDH in four each of healthy control and patients were determined by SYBR Green based qRT-PCR. Data is expressed as mean \pm SD from three independent experiments. $* * p<0.01$, *** $p<0.001$, and $* * * * p<0.0001$. (C) Different single amino acid substitutions in P1 and P4 impeded the PYCR1 half-life as determined by cycloheximide (CHX) chase assay. Cells were harvested and lysed at $0,4,8,16$, and $24 \mathrm{~h}$ after treatment in medium containing $20 \mu \mathrm{g} / \mathrm{ml} \mathrm{CHX}$. (D) Results in $(\mathbf{C})$ were quantified using the IMAGE J software. $\alpha$-tubulin was used to normalize the PYCR1 protein levels. The relative levels at $0 \mathrm{hr}$ were defined as 1 for each sample.
\end{abstract}

\section{Expression of genes encoding ECM components was reduced in fibroblasts with PYCR1 mutations}

RNA-Seq transcriptome analysis of two patients (P1 and P3) and two controls detected approximately 60.8 to 75.9 million read pairs per sample, which were unambiguously mapped to 21,141 reference gene sequences (NCBI Build 37/hg19 database; total 23,769 human genes). Among the putative differentially expressed genes with at least 2-fold change and a minimum of 10 TPM in patients or controls, sequence annotations indicated the proteins of $17(53.1 \%)$ of the genes downregulated and $2(9.0 \%)$ of the the genes upregulated in patients are localized in the extracellular space. Expression changes of the most downregulated gene periostin (POSTN) and the most upregulated gene homeobox B7 (HOXB7) (Table 2 and Fig. 3A) in fibroblasts from patients were confirmed through SYBR Green-based qRT-PCR (Fig. 3B).
Gene Ontology analysis of the differentially expressed candidates for cellular component distributions using the PANTHER Classification System indicated ECM and extracellular region proteins were enriched in patients with PYCRI mutations (www.pantherdb.org/). A biased distribution of essentially downregulated candidates of ECM and extracellular protein genes was found (Fig. 3C). These included genes encoding the ECM/extracellular region proteins POSTN, metallopeptidase 1 (MMP1), insulin-like growth factorbinding protein 3 (IGFBP3), ELN, complement C1s (C1S), ADAM metallopeptidase with thrombospondin type 1 motif 5 (ADAMTS5), and vascular endothelial growth factor A (VEGFA).

Among the aforementioned proteins, the downregulated ECM components, elastin (ELN) and decorin (DCN), are associated with different phenotypes of aging, such as cutis laxa and joint and dermal manifestations $[3,19]$. In addition to these two proteins, 
the mRNA levels of seven other downregulated extracellular space proteins, POSTN, MMP1, IGFBP3, pentraxin 3 (PTX3), EMILIN2, thrombospondin 2 (THBS2), and vascular endothelial growth factor A (VEGFA), and the upregulated ECM protein pleiotrophin (PTN) were quantified using qRT-PCR in a different set of controls and skin fibroblasts from all four patients (Fig.
3D). Of the 10 examined genes, MMP1 was only downregulated in patients $\mathrm{P} 1, \mathrm{P} 2$, and $\mathrm{P} 3$, but not in $\mathrm{P} 4$, and $P T N$ did not show significant upregulation in patient P1. Hence, MMP1 and PTN were excluded for further analysis.
A

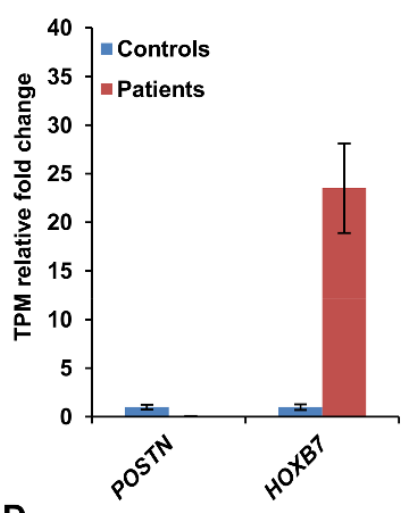

B

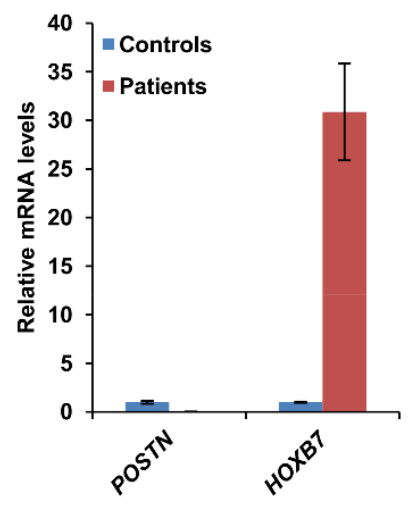

C

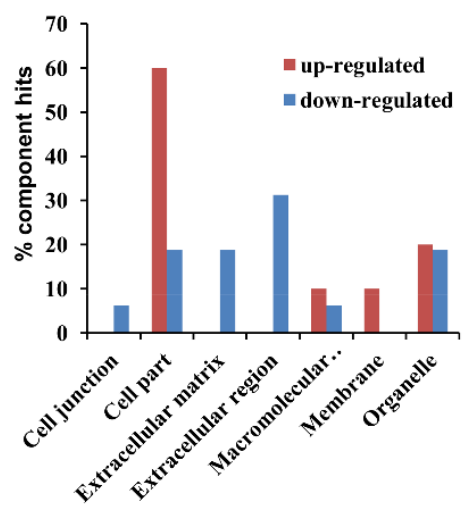

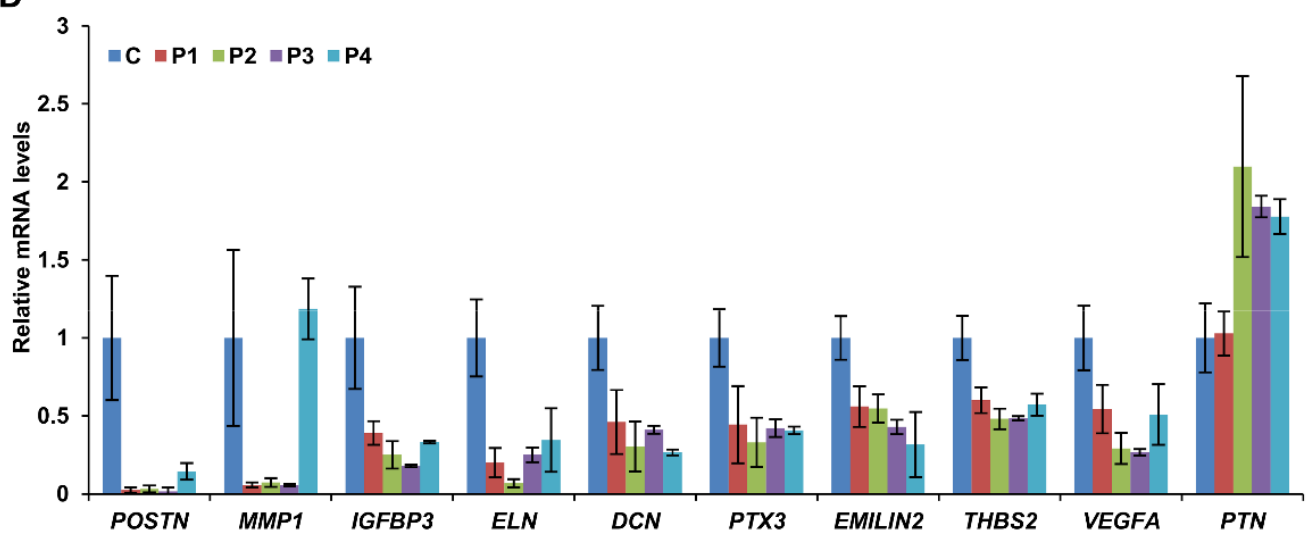

Figure 3. qRT-PCR validated the expression changes of PYCRI affected extracellular space protein genes. (A) Relative change of the most up- and downregulated expressed genes as determined by RNA-Seq analysis. (B) qRT-PCR validation of the two candidates in (A). (C) Gene ontology (GO) analysis shows enrichment of genes related to extracellular marix/region components are downregulated in patients' fibroblasts. (D) The expression levels of ten extracellular space protein genes (fold-change $\geq 2$, TPM $\geq 10$ ) in the skin fibroblasts of all four patients and three different healthy donors were quantified by qRT-PCR. Data is expressed as mean \pm SD from three independent experiments. * $p<0.05$, ** $p<0.01$.

\section{Association of diseases and functions revealed by Ingenuity Pathway Analysis}

To explore the diseases and functions that may be related to PYCRI mutations, 54 differentially expressed gene candidates with $T P M \geq 10$ and with fold changes $\geq 2$ were analyzed using the Ingenuity Pathway Analysis (IPA) software. With the pertinent parameter settings, IPA of "Diseases and disorders" and "Physiological system development and function" revealed these genes in 16 modules $(p<0.05)$ (Fig. 4A). The top five potentially affected modules were related to the cardiovascular system, ophthalmic disease, dermatological diseases, and neurological disease, and each contained at least five downregulated extracellular space genes (Fig. 4B). Among the differentially expressed genes, the mRNA levels of DCN, EMILIN2, IGFBP3, MMP1, PTX3, $T H B S 2$, and $V E G F A$ was quantified using qRT-PCR (Fig. 3D). 


\section{Perturbation of PYCR1 affected ECM gene expression}

RNA-Seq analysis revealed the expression of several ECM genes and genes of other categories were altered in the primary cultures of fibroblasts from ARCL2B patients. The most downregulated gene in fibroblasts from patients in RNA-Seq analysis was POSTN. The expression of the POSTN protein was also decreased in fibroblasts from the four patients (Fig. 5A). The correlation between the PYCR1 mutation and ECM gene expression was further evaluated by perturbing the PYCR1 expression in fibroblasts from controls and patients.

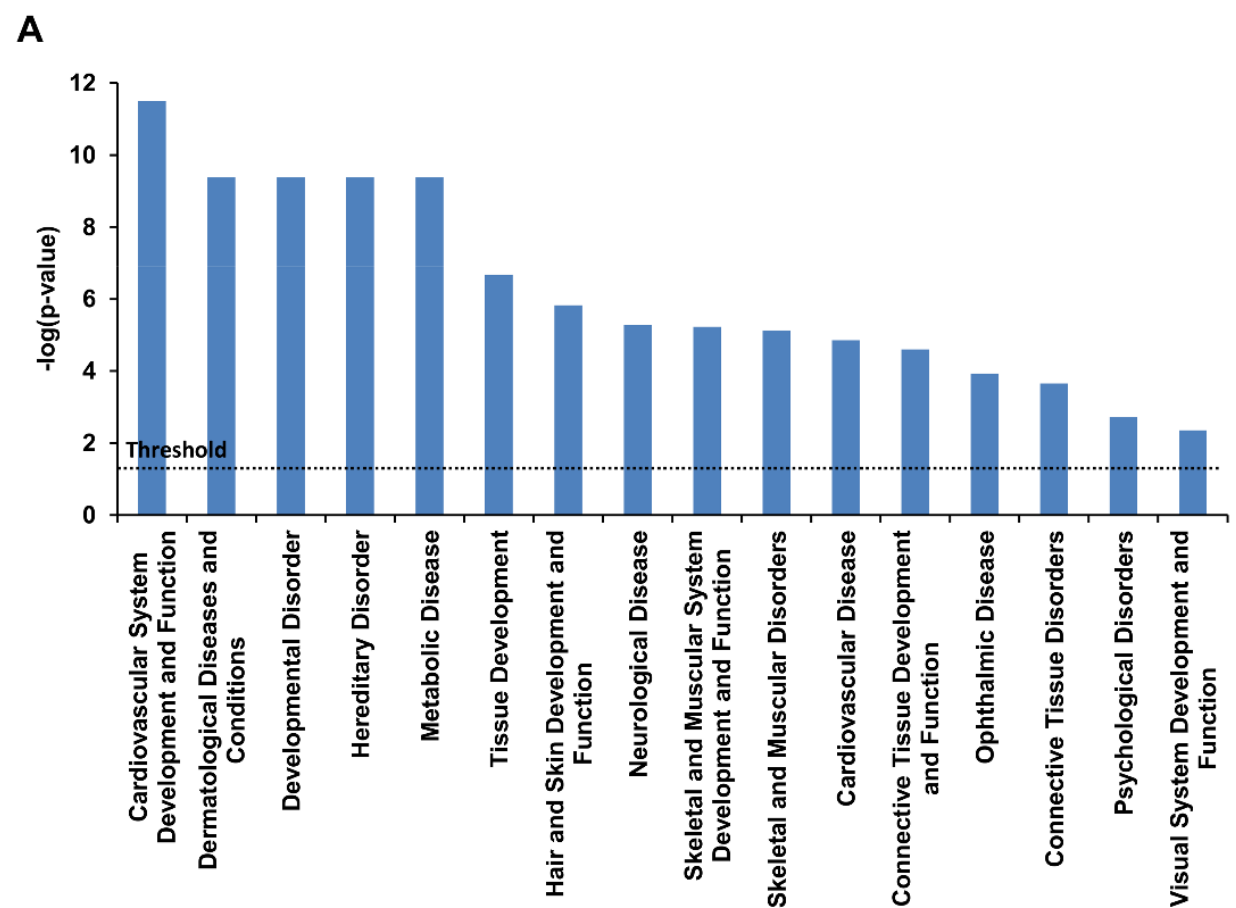

B

\begin{tabular}{|c|c|}
\hline Category & Molecules \\
\hline Cardiovascular Disease & 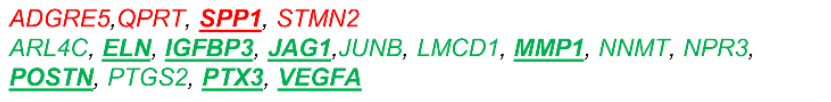 \\
\hline Ophthalmic Disease & $\begin{array}{l}\text { QPRT, } \underline{S P P 1} \\
C Y P 1 B 1, \underline{D C N}, \underline{E F E M P 1}, \underline{F B L N 2}, \underline{J A G 1}, \text { NNMT, PTGS2, VEGFA }\end{array}$ \\
\hline $\begin{array}{l}\text { Dermatological Diseases } \\
\text { and Conditions }\end{array}$ & 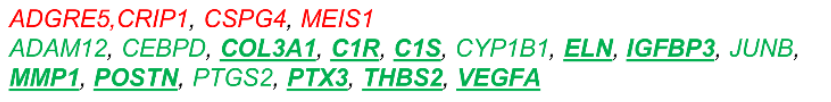 \\
\hline Neurological Disease & $\begin{array}{l}\text { ADGRE5, CSPG4, QPRT, SPP1 } \\
\text { ADAM12, } \underline{A D A M T S 5}, A R L 4 C, C E B P D, \underline{C O L 3 A 1}, \underline{E L N}, \underline{E F E M P 1}, \underline{\text { IGFBP3 }}, \\
\underline{\text { JAG1}}, J U N B, L M C D 1, N N M T, P T G S 2, \underline{P T X 3}, \underline{\text { VEGFA }}\end{array}$ \\
\hline $\begin{array}{l}\text { Cardiovascular System } \\
\text { Development and Function }\end{array}$ & $\begin{array}{l}\text { ADGRE5, AMIGO2, } \underline{P T N} \\
\underline{D C N}, \underline{F B L N 2}, \underline{\text { IGFBP3}}, \underline{T H B S 2}, \text { PTGS2, } \underline{P T X 3}, \underline{\text { VEGFA }}\end{array}$ \\
\hline
\end{tabular}

Figure 4. IPA analysis indicated differentially expressed gene candidates are associated with cardiovascular, ophthalmic, and dermatological diseases. Genes with fold change $\geq 2$ and TPM $>$ 10 were analyzed. (A) Genes implicated in the principal biological function categories related to the catalogues "Diseases and Disorders" and "Physiological System Development and Function". (B) List of the genes implicated in top five catalogues. The input putatively up- and downregulated genes are indicated by red and green texts, respectively. The molecules located in extracellular space are bold and underlined. 
A
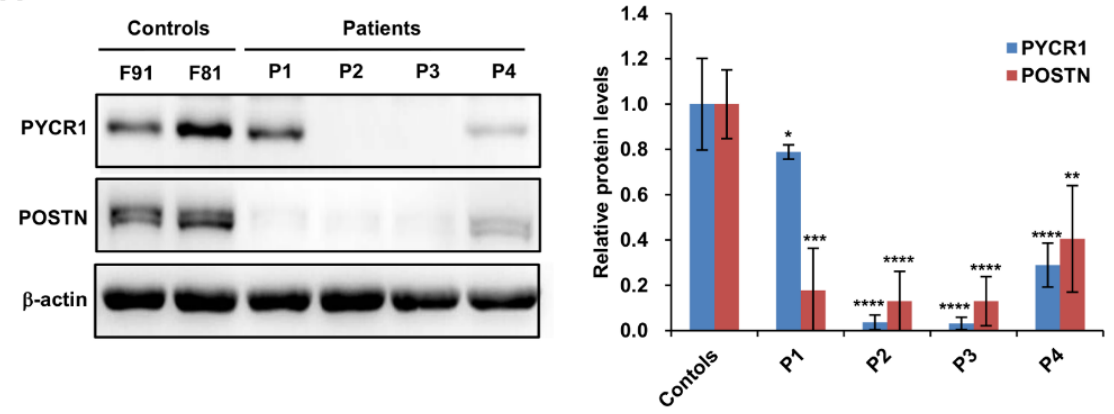

B

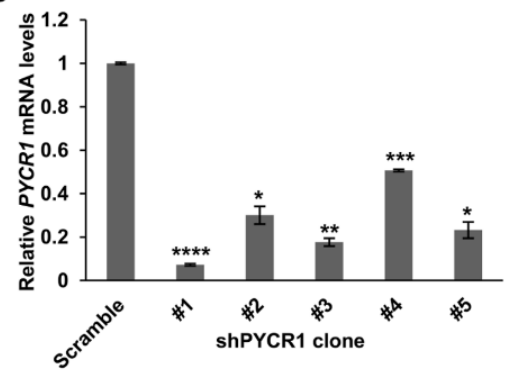

C

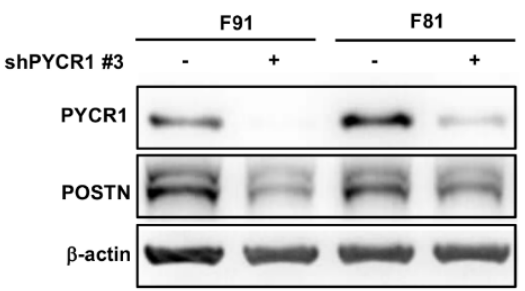

D

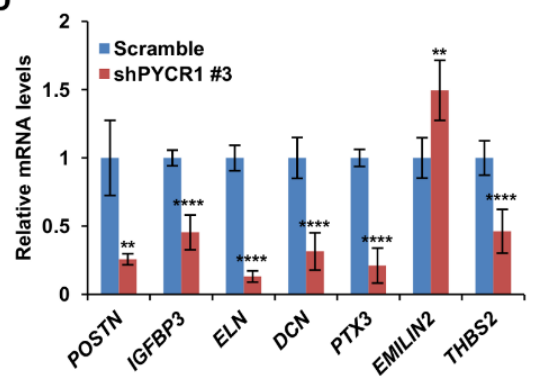

E

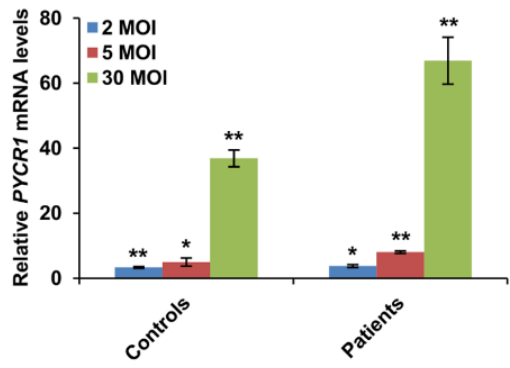

$\mathbf{F}$

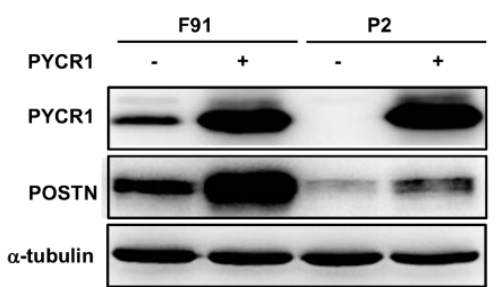

G

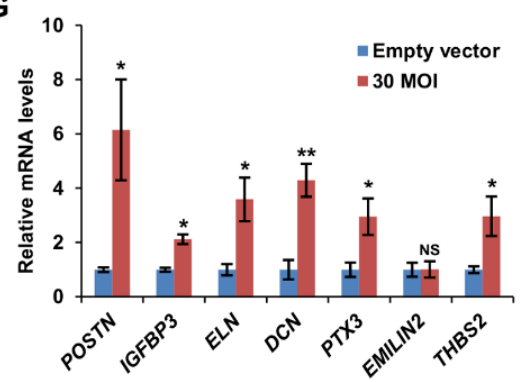

Figure 5. Perturbations of PYCR1 expression modulate the expression of extracellular space genes in skin fibroblast. (A) Western blot (left) and quantification (right) of PYCR1 and POSTN protein levels in primary skin fibroblasts from controls and patients. Data was expressed as means \pm SD from four biological replicates. (B) Evaluation of knockdown efficiencies of pLKO.1 lentiviruses expressing five independent PYCRI shRNAs in primary skin fibroblasts. At $48 \mathrm{~h}$ post-transduction, skin fibroblasts were collected for RNA isolation and qRT-PCR. (C) At $72 \mathrm{~h}$ post-transduction, proteins were isolated from fibroblasts and analyzed by Western blot. Scramble shRNA was used as negative control. (D) Knockdown of PYCR1 (shPYCR1 \#3, 2MOI) in skin fibroblast modulates the expression of extracellular space genes. (E) Evaluation of the overexpression of PYCRI in human fibroblasts infected with 2, 5, and $30 \mathrm{MOI}$ of recombinant PYCRl lentiviruses relative to the empty vector control. (F) Western blot analyses revealed that PYCR1 overexpression (30 MOI) induce POSTN expression in skin fibroblast cells. (G) PYCRl overexpression in patient's skin fibroblasts (P2) modulates expression of extracellular space genes. Data was expressed as means \pm SD from three biological replicates. NS: not significant, $* p<0.05$, $* * p<0.01$, *** $p<0.001$, and $* * * * p<0.0001$. 
Modulation of PYCR1 expression via shRNA knockdown or overexpression affected the expression of almost all analyzed extracellular space genes. Transduction of skin fibroblasts with five $P Y C R I$-specific shRNAs reduced PYCRI expression from $50 \%$ to $93 \%$ (Fig. 5B) and the expression of POSTN, the most downregulated gene in ARCL2B patients as detected by RNA-Seq, from $0 \%$ to $75 \%$ (Supplementary Fig. 1A). The data indicated that POSTN expression in skin fibroblasts was correlated with the PYCRI expression level $\left(\mathrm{R}^{2}=\right.$ 0.9143), and that shPYCR1 \#1 had the highest knockdown efficiency in PYCRI and POSTN (93\% and $75 \%$, respectively). However, knockdown with shPYCR1 \#1 also partly affected the expression of PYCR2 (Supplementary Fig. 2A), most likely due to the high homology of target sequences in PYCR1 and PYCR2 mRNAs [20] (Supplementary Fig. 2B). Thus, instead of shPYCR1 \#1, shPYCR1 \#3 was chosen for further analysis because it had minor influence on PYCR2 expression. Western blot analysis also showed the downregulation of POSTN expression (Fig. 5C). Figure 5D shows the qRT-PCR expression profiling results, which indicated the reduced expression of extracellular space genes, including POSTN, IGFBP3, ELN, DCN, $P T X 3$, and THBS2, but not EMILIN2, in PYCR1 knockeddown cells. In contrast to PYCRI knockdown, PYCRI overexpression in fibroblasts from patients not only restored the intracellular proline level (Supplementary Fig. 3) to a higher level but also increased the mRNA and protein expression levels of POSTN and most of the other extracellular space genes (Fig. 5F and 5G). These results indicate that PYCR1 modulates the expression of extracellular space genes, except for EMILIN2.

\section{DISCUSSION}

In this study, we provided the first characterization of the transcriptome for PYCRI mutations causing the neurocutaneous syndrome ARCL2B. This study also revealed previously unknown associations between the PYCR1 mutation and molecular changes in patients with ARCL2B. In our patients, PYCR1 deficiency certainly led to a premature aging phenotype, but this is the first report of heart valves disease and preglaucoma in such patients. No effective therapeutic strategies are yet available for ARCL2B, and the underlying mechanisms involved in disease-related changes require further elucidation.

Diverse PYCRI mutations led to different half-lives of the resulting PYCR1 proteins. The PYCR1 mutant protein with the G248E substitution showed slower degradation than that with the A187T substitution but resulted in with more severe neurological and orthopedic manifestations, thereby suggesting that p.G248E is null at the functional level [11]. Because PYCR1 catalyzes the conversion of $\mathrm{P} 5 \mathrm{C}$ to proline, PYCRI mutations are expected to lead to decreased intracellular proline levels. To confirm this, we also showed that PYCR1 overexpression increased intracellular proline levels. Hence, PYCR1 modulates proline levels in skin fibroblasts. No significant difference was observed in the intracellular proline level between patients with homozygous frameshift (P2 and P3) and compound heterozygous frameshift and missense mutations (P1 and P4). These patients only showed the truncated protein p.P115fsX7 and the truncated plus mutant protein with the G248E or A187T substitution. The results differed from those of Reversade et al. [5], probably due to differences in culture conditions and the measurement method. Proline and its derivative, hydroxyproline, play an essential role in the formation of collagen and elastin, which are major ECM components in many organs, such as the connective tissue. Decreases in proline levels may affect collagen and elastin synthesis in connective tissues, leading to cutis laxa [2].

Transcriptome analysis of the fibroblasts from patients' with PYCRI mutations and controls revealed that many of the differentially expressed genes coded for proteins localized in the extracellular space. Genetic mutations in these proteins are associated with different phenotypes of aging. Many ECM components are found to be decreased in patients with cutaneous syndromes because alterations of ECM composition may lead to abnormal elastic fibers, thus causing cutis laxa [3]. In addition to providing structural and biochemical support to the surrounding cells, ECM also offers a dynamic and complex environment for interactions with other ECM components. Cell-matrix interactions not only mediate adhesion but also transduce signals to modulate cell survival, proliferation, differentiation, phenotype, and behavior [21]. Therefore, alterations of ECM composition during development affect cell behaviors, leading to ECM dysregulation and disease progression [22]. Furthermore, in the present study, IPA revealed that the affected genes associated with cardiovascular disease as one of the main aging-associated diseases; these genes included the genes for many extracellular space protein $(D C N, E L N$, IGFBP3, POSTN, PTX3, THBS1, and THBS2), which were downregulated in fibroblasts from ARCL2B patients. POSTN, the most downregulated gene in affected individuals, is a heterofunctional regulator of cardiac development and disease [23, 24]. Our study demonstrated for the first time that many extracellular space genes (POSTN, IGFBP3, ELN, DCN, PTX3, and $T H B S 2$ ) are affected by PYCR1. This study also provided a comprehensive linkage of extracellular space genes for 
the neurocutaneous syndrome caused by PYCRI mutations.

We demonstrated that POSTN expression in skin fibroblasts is correlated with the PYCRI expression level and is significantly decreased in adult skin fibroblasts (Supplementary Fig. 1). POSTN comprises an aminoterminal EMI domain, four repeated domains related to those found in fasciclin-1 (fasciclin-1-like repeats), and a carboxyl-terminal domain. It belongs to the family of fasciclins based on its homology to fasciclin 1 (FAS1), an insect cell adhesion protein involved in CNS development. POSTN is expressed not only during embryogenesis or pathogenesis [25, 26] (including neoplasia, tissue repair, and cardiac injury) but also in various organs, particularly fibroblast-rich connective tissues such as the skin or breast [27]. POSTN has pivotal functions in heart valvulogenesis [28-30], trabecular meshwork development [31], skin wound healing [32], brain and CNS development [33, 34], and skin aging [35]. However, the role of POSTN in neurocutaneous syndrome has never been described. Studies have shown that $C Y P 1 B 1$ is a causative gene in primary congenital glaucoma [36, 37], and that the phenotype of Cyp $1 b 1^{-1-}$ mouse model resembled glaucomatous human eyes [31].

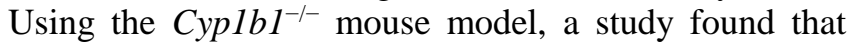
CYP1B1 deficiency increased oxidative stress and decreased POSTN production, leading to trabecular meshwork tissue dysgenesis and dysfunction [31]. We also found that PYCR1 modulated CYP1B1 expression in skin fibroblasts (Supplementary Fig. 4). Taken together, POSTN may play a crucial role in skin aging, heart valve disease, and preglaucoma.

PYCR1 deficiency also leads to irregular, fragmented, and reduced elastic fibers [5, 6]. Our study found that $E L N$ expression might be regulated by PYCR1. ELN is a major component of ECM; thus, its loss will affect ECM assembly. Expression of microfibril and elastic fiber-associated molecules such as DCN, EMILIN2 (Fig. 3D), TGFBI, FBLN2, and FBLN3 (EFEMP1) (Supplementary Fig. 5) were also reduced in fibroblasts from ARCL2B patients. These molecules provide structural support to organs and tissues such as the heart, skin, lungs, ligaments, and blood vessels. These findings suggested that PYCR1 deficiency caused the loose arrangement of elastic fibers and the presence of fine and short fragmented microfibrils.

CRIP2, which plays a crucial role in atrioventricular valve development [38] and coronary smooth muscle differentiation [39], was one of the most upregulated genes in affected individuals, but no significant change in PYCRl knockdown or overexpression was found (Supplementary Fig. 6). The result suggests that the upregulation of CRIP2 in skin fibroblasts from affected individuals is not modulated by PYCR1 and may be a dosage compensation effect. Furthermore, our data also indicate that PTN is not modulated by PYCR1. PTN is a secreted cell signaling growth factor and developmentregulated cytokine, also known as osteoblast-specific factor 1 (OSF-1); it has multiple effects on cell proliferation, differentiation, and angiogenesis. It becomes re-activated in response to injury. PTN had been reported to have roles in bone [40] and heart injury repair [41] and as a neuromodulator [42]. A previous study found that PTN was upregulated in the heart of Postn ${ }^{-/}$ mice, and that POSTN suppressed PTN expression [43]. This suggests that our findings may be extendable to the human disease.

There are two paralogs of PYCR1 in humans - the highly similar PYCR2 (84\% homology) and the more distantly related PYCRL (45\% homology to the other two forms). Both PYCR1 and PYCR2 are thought to localize to the mitochondria and primarily catalyze the conversion of glutamate to proline, and PYCRL is a cytoplasmic enzyme [44]. A study indicated no significant changes in PYCR2 expression in fibroblasts from individuals with PYCR1 mutations [5]. We did not observe the dosage compensation of PYCR2 and PYCRL in affected individuals (Supplementary Fig. 7). Recently, Nakayama et al. showed that PYCR2 mutations caused microcephaly and hypomyelination in humans [45]. The different phenotypes caused by the PYCR 1 and $P Y C R 2$ variants and their different expression levels in various tissues suggest that each of these isozymes has a unique role in the human body.

In conclusion, we found that PYCRI mutations significantly reduced the expression of many extracellular space genes (POSTN, IGFBP3, ELN, DCN, PTX3, and THBS2), and that PYCR1 may modulate the expression of these extracellular space genes in skin fibroblasts. ELN mutations resulting in autosomal-dominant cutis laxa are known to be associated with different phenotypes of aging (cutis laxa and cardiovascular and musculoskeletal abnormalities), and the $D C N$ mutation leads to abnormal collagen fibril morphology and skin fragility [3, 46, 47]. Most importantly, we found a novel candidate POSTN, which was the major downregulated gene in affected individuals and the expression of which in skin fibroblasts cells was correlated with PYCR1 expression. POSTN has never been described in PYCR1-related ARCL, and our results indicate that POSTN may play an important role in skin aging, heart valves disease, and preglaucoma.

\section{Acknowledgments}

The authors thank the patients and healthy donors for their participation in this study, National RNAi Core Facility at 
Academia Sinica in Taiwan for providing shRNA reagents and related services, and Wallace Academic Editing for proofreading and editing the manuscript. This work was supported by the Ministry of Science and Technology [MOST-105-2314-B-195-011-MY2] and grants from Mackay Memorial Hospital [MMH-E-10402, MMH-E-105-02, MMH-E-106-02, МMH-CT-10410, MMH-CT-10510], Taipei, Taiwan.

\section{Conflicts of interest}

The author declares no conflicts of interest.

\section{Supplementary data}

Supplementary data is available online at www.aginganddisease.org/EN/10.14336/AD.2018.0222

\section{References}

[1] Morava E, Guillard M, Lefeber DJ, Wevers RA (2009). Autosomal recessive cutis laxa syndrome revisited. Eur J Hum Genet, 17: 1099-1110.

[2] Mohamed M, Kouwenberg D, Gardeitchik T, Kornak U, Wevers RA, Morava E (2011). Metabolic cutis laxa syndromes. J Inherit Metab Dis, 34: 907-916.

[3] Berk DR, Bentley DD, Bayliss SJ, Lind A, Urban Z (2012). Cutis laxa: a review. J Am Acad Dermatol, 66: 842 e841-817.

[4] Uitto J, Li Q, Urban Z (2013). The complexity of elastic fibre biogenesis in the skin--a perspective to the clinical heterogeneity of cutis laxa. Exp Dermatol, 22: 88-92.

[5] Reversade B, Escande-Beillard N, Dimopoulou A, Fischer B, Chng SC, Li Y, et al. (2009). Mutations in PYCR1 cause cutis laxa with progeroid features. Nat Genet, 41: 1016-1021.

[6] Kretz R, Bozorgmehr B, Kariminejad MH, Rohrbach M, Hausser I, Baumer A, et al. (2011). Defect in proline synthesis: pyrroline-5-carboxylate reductase 1 deficiency leads to a complex clinical phenotype with collagen and elastin abnormalities. J Inherit Metab Dis, 34: 731-739.

[7] Baumgartner MR, Hu CA, Almashanu S, Steel G, Obie C, Aral B, et al. (2000). Hyperammonemia with reduced ornithine, citrulline, arginine and proline: a new inborn error caused by a mutation in the gene encoding delta(1)pyrroline-5-carboxylate synthase. Hum Mol Genet, 9: 2853-2858.

[8] Adams E, Frank L (1980). Metabolism of proline and the hydroxyprolines. Annu Rev Biochem, 49: 1005-1061.

[9] Dimopoulou A, Fischer B, Gardeitchik T, Schroter P, Kayserili H, Schlack C, et al. (2013). Genotypephenotype spectrum of PYCRl-related autosomal recessive cutis laxa. Mol Genet Metab, 110: 352-361.

[10] Guernsey DL, Jiang H, Evans SC, Ferguson M, Matsuoka M, Nightingale M, et al. (2009). Mutation in pyrroline-5-carboxylate reductase 1 gene in families with cutis laxa type 2. Am J Hum Genet, 85: 120-129.

[11] Lin DS, Chang JH, Liu HL, Wei CH, Yeung CY, Ho CS, et al. (2011). Compound heterozygous mutations in PYCR1 further expand the phenotypic spectrum of De Barsy syndrome. Am J Med Genet A, 155A: 3095-3099.

[12] Lin DS, Yeung CY, Liu HL, Ho CS, Shu CH, Chuang CK, et al. (2011). A novel mutation in PYCRI causes an autosomal recessive cutis laxa with premature aging features in a family. Am J Med Genet A, 155A: 12851289.

[13] Yildirim Y, Tolun A, Tuysuz B (2011). The phenotype caused by PYCRl mutations corresponds to geroderma osteodysplasticum rather than autosomal recessive cutis laxa type 2. Am J Med Genet A, 155A: 134-140.

[14] Scherrer DZ, Baptista MB, Matos AH, Maurer-Morelli CV, Steiner CE (2013). Mutations in PYCRl gene in three families with autosomal recessive cutis laxa, type 2. Eur J Med Genet, 56: 336-339.

[15] Mi H, Muruganujan A, Thomas PD (2013). PANTHER in 2013: modeling the evolution of gene function, and other gene attributes, in the context of phylogenetic trees. Nucleic Acids Res, 41: D377-386.

[16] Thomas PD, Campbell MJ, Kejariwal A, Mi H, Karlak B, Daverman R, et al. (2003). PANTHER: a library of protein families and subfamilies indexed by function. Genome Res, 13: 2129-2141.

[17] Wang SH, Liu M, Chi MG, Wang QD, Sun MJ (2004). Production of human liver prolidase by Saccharomyces cerevisiae as host cells. Acta Pharmacol Sin, 25: 794800.

[18] Colan SD, McElhinney DB, Crawford EC, Keane JF, Lock JE (2006). Validation and re-evaluation of a discriminant model predicting anatomic suitability for biventricular repair in neonates with aortic stenosis. J Am Coll Cardiol, 47: 1858-1865.

[19] Nareyeck G, Seidler DG, Troyer D, Rauterberg J, Kresse H, Schonherr E (2004). Differential interactions of decorin and decorin mutants with type I and type VI collagens. Eur J Biochem, 271: 3389-3398.

[20] Kuo ML, Lee MB, Tang M, den Besten W, Hu S, Sweredoski MJ, et al. (2016). PYCR1 and PYCR2 Interact and Collaborate with RRM2B to Protect Cells from Overt Oxidative Stress. Sci Rep, 6: 18846.

[21] Gattazzo F, Urciuolo A, Bonaldo P (2014). Extracellular matrix: a dynamic microenvironment for stem cell niche. Biochim Biophys Acta, 1840: 2506-2519.

[22] Freedman BR, Bade ND, Riggin CN, Zhang S, Haines PG, Ong KL, et al. (2015). The (dys)functional extracellular matrix. Biochim Biophys Acta, 1853: 31533164.

[23] Kuhn B, del Monte F, Hajjar RJ, Chang YS, Lebeche D, Arab S, et al. (2007). Periostin induces proliferation of differentiated cardiomyocytes and promotes cardiac repair. Nat Med, 13: 962-969.

[24] Conway SJ, Molkentin JD (2008). Periostin as a heterofunctional regulator of cardiac development and disease. Curr Genomics, 9: 548-555. 
[25] Hamilton DW (2008). Functional role of periostin in development and wound repair: implications for connective tissue disease. J Cell Commun Signal, 2: 917.

[26] Frangogiannis NG (2012). Matricellular proteins in cardiac adaptation and disease. Physiol Rev, 92: 635688.

[27] Tilman G, Mattiussi M, Brasseur F, van Baren N, Decottignies A (2007). Human periostin gene expression in normal tissues, tumors and melanoma: evidences for periostin production by both stromal and melanoma cells. Mol Cancer, 6: 80.

[28] Kruzynska-Frejtag A, Machnicki M, Rogers R, Markwald RR, Conway SJ (2001). Periostin (an osteoblast-specific factor) is expressed within the embryonic mouse heart during valve formation. Mech Dev, 103: 183-188.

[29] Lindner V, Wang Q, Conley BA, Friesel RE, Vary CP (2005). Vascular injury induces expression of periostin: implications for vascular cell differentiation and migration. Arterioscler Thromb Vasc Biol, 25: 77-83.

[30] Ghatak S, Misra S, Norris RA, Moreno-Rodriguez RA, Hoffman S, Levine RA, et al. (2014). Periostin induces intracellular cross-talk between kinases and hyaluronan in atrioventricular valvulogenesis. J Biol Chem, 289: 8545-8561.

[31] Zhao Y, Wang S, Sorenson CM, Teixeira L, Dubielzig RR, Peters DM, et al. (2013). Cyp1b1 mediates periostin regulation of trabecular meshwork development by suppression of oxidative stress. Mol Cell Biol, 33: 42254240.

[32] Jackson-Boeters L, Wen W, Hamilton DW (2009). Periostin localizes to cells in normal skin, but is associated with the extracellular matrix during wound repair. J Cell Commun Signal, 3: 125-133.

[33] Shimamura M, Taniyama Y, Katsuragi N, Koibuchi N, Kyutoku M, Sato N, et al. (2012). Role of central nervous system periostin in cerebral ischemia. Stroke, 43: 11081114.

[34] Ma SM, Chen LX, Lin YF, Yan H, Lv JW, Xiong M, et al. (2015). Periostin Promotes Neural Stem Cell Proliferation and Differentiation following HypoxicIschemic Injury. PLoS One, 10: e0123585.

[35] Egbert M, Ruetze M, Sattler M, Wenck H, Gallinat S, Lucius R, et al. (2014). The matricellular protein periostin contributes to proper collagen function and is downregulated during skin aging. J Dermatol Sci, 73: 4048.

[36] Panicker SG, Reddy AB, Mandal AK, Ahmed N, Nagarajaram HA, Hasnain SE, et al. (2002). Identification of novel mutations causing familial primary congenital glaucoma in Indian pedigrees. Invest Ophthalmol Vis Sci, 43: 1358-1366.
[37] Vincent AL, Billingsley G, Buys Y, Levin AV, Priston M, Trope G, et al. (2002). Digenic inheritance of earlyonset glaucoma: CYP1B1, a potential modifier gene. Am J Hum Genet, 70: 448-460.

[38] Kim JD, Kim HJ, Koun S, Ham HJ, Kim MJ, Rhee M, et al. (2014). Zebrafish Crip2 plays a critical role in atrioventricular valve development by downregulating the expression of ECM genes in the endocardial cushion. Mol Cells, 37: 406-411.

[39] Chang DF, Belaguli NS, Iyer D, Roberts WB, Wu SP, Dong XR, et al. (2003). Cysteine-rich LIM-only proteins CRP1 and CRP2 are potent smooth muscle differentiation cofactors. Dev Cell, 4: 107-118.

[40] Lamprou M, Kaspiris A, Panagiotopoulos E, Giannoudis PV, Papadimitriou E (2014). The role of pleiotrophin in bone repair. Injury, 45: 1816-1823.

[41] Li J, Wei H, Chesley A, Moon C, Krawczyk M, Volkova $\mathrm{M}$, et al. (2007). The pro-angiogenic cytokine pleiotrophin potentiates cardiomyocyte apoptosis through inhibition of endogenous AKT/PKB activity. J Biol Chem, 282: 34984-34993.

[42] Gonzalez-Castillo C, Ortuno-Sahagun D, GuzmanBrambila C, Pallas M, Rojas-Mayorquin AE (2014). Pleiotrophin as a central nervous system neuromodulator, evidences from the hippocampus. Front Cell Neurosci, 8: Article 443.

[43] Tkatchenko TV, Moreno-Rodriguez RA, Conway SJ, Molkentin JD, Markwald RR, Tkatchenko AV (2009). Lack of periostin leads to suppression of Notch1 signaling and calcific aortic valve disease. Physiol Genomics, 39: 160-168.

[44] De Ingeniis J, Ratnikov B, Richardson AD, Scott DA, Aza-Blanc P, De SK, et al. (2012). Functional specialization in proline biosynthesis of melanoma. PLoS One, 7: e45190.

[45] Nakayama T, Al-Maawali A, El-Quessny M, Rajab A, Khalil S, Stoler JM, et al. (2015). Mutations in PYCR2, Encoding Pyrroline-5-Carboxylate Reductase 2, Cause Microcephaly and Hypomyelination. Am J Hum Genet, 96: 709-719.

[46] Hansen NU, Genovese F, Leeming DJ, Karsdal MA (2015). The importance of extracellular matrix for cell function and in vivo likeness. Exp Mol Pathol, 98: 286294.

[47] Danielson KG, Baribault H, Holmes DF, Graham H, Kadler KE, Iozzo RV (1997). Targeted disruption of decorin leads to abnormal collagen fibril morphology and skin fragility. J Cell Biol, 136: 729-743. 